\title{
MARKOV RELIABILITY MODELS OF SERIES SYSTEMS WITH REDUNDANCY AND REPAIR FACILITIES
}

\author{
Yuriy Zhernovyi ${ }^{1}$, Bohdan Kopytko ${ }^{2}$ \\ ${ }^{1}$ Ivan Franko National University of Lviv, Lviv, Ukraine \\ ${ }^{2}$ Department of Mathematics, Czestochowa University of Technology \\ Czestochowa, Poland \\ yuriy.zhernovyy@lnu.edu.ua,bohdan.kopytko@im.pcz.pl
}

Received: 08 August 2021; Accepted: 09 September 2021

\begin{abstract}
In this paper, we use Markov models for studying the reliability of series systems with redundancy and repair facilities. We suppose that the units' time to failure and recovery times are exponentially distributed. We consider the cases when $1 \leq c \leq m$ and $m+1 \leq c \leq m+n$, for the system of $n$ operating units, $m$ unloaded redundant units and $c$ repair facilities. Using the exponential distributions properties, we obtain stationary reliability indices of the series systems: steady-state probabilities, a stationary availability coefficient, mean time to failure, mean time between failures and mean downtime.
\end{abstract}

MSC 2010: 60G10, 60J28, 60K25, $93 B 40$

Keywords: reliability, recoverable system, redundancy, series system, Markov models, mean time to failure, mean time between failures, mean downtime

\section{Introduction}

Series systems of identical independent units with a common group of redundant units are common in engineering practice. Formally, such a structure appears if a system consists of units of several types [1,2]. A set of units of the same type can be considered as a "series system", for which there is a stock of spare units. It is reasonable to consider these spare units as unloaded; these units wait for being switched into operating position after one of the operating units has failed. Failed units are directed to a repair shop from where, after recovery, they again enter the system's stock. The switching of a spare unit into an operating position is usually assumed instantaneous.

Recoverable series systems differ by their recovery processes. Assume that during recovering a currently failed unit system continues to stay in an operational state, so new failures may appear. In principle, in this case one can observe even a situation when all system units have failed. It can happen if, for instance, a recovery process is very slow. 
Consider a system that consists of $r=m+n$ identical units, namely, $n$ main operating units and $m$ unloaded redundant units. The system stops functioning in normal mode at the moment when the number of failed units reaches $m+1$. Assume that those $n-1$ units, which were serviceable at the moment of system failure in idle time during recovery, continue to operate and may fail. In addition, the number $c$ of repair facilities can be restricted $(c \leq r)$, so failed units can form a queue for recovering.

If we consider the described system as a queueing system, then in the absence of redundant units $(m=0)$, it is a classical closed queueing system [3]. The closed system is also known as a system with a finite number of sources or the Engset system.

The method of potentials was used in [4] to construct an algorithm that makes it possible to calculate the steady-state distribution of the number of failed units for a single-channel closed system with an exponential distribution of the units' time to failure and an arbitrary distribution of recovery time. This method is not suitable for arbitrary distributions of the units' time to failure.

A method for calculating steady-state probability distributions of the multichannel closed queueing systems with exponential distribution of the time to failure and an arbitrary distribution of recovery time is proposed in [5].

In most academic approaches, a random time to failure and a random recovery time are assumed exponentially distributed for all units that give a possibility to use the Markov model for a reliability study. In work [1], steady-state probabilities $p_{k}$ of having $k$ failed units were found for the case when $c=m+n$. In this paper, we consider the cases when $1 \leq c \leq m$ and $m+1 \leq c \leq m+n$, and we obtain stationary reliability indices of the series systems: steady-state probabilities, stationary availability coefficient, mean time to failure, mean time between failures and mean downtime.

Let $p_{k}$ be the steady-state probability of having $k$ failed units, then the stationary reliability indices of the system are determined by the formulas:

$$
K=\sum_{k=0}^{m} p_{k}, \quad N=\sum_{k=1}^{r} k p_{k} .
$$

Here, $K$ is the stationary availability coefficient, and $N$ is the average number of failed units.

\section{Stationary reliability indices of the series systems}

Let us denote states by natural numbers $0,1,2, \ldots$, where the number of a state corresponds to the number of failed units. Then the state graphs have the form shown in Figures 1 and 2, respectively. 


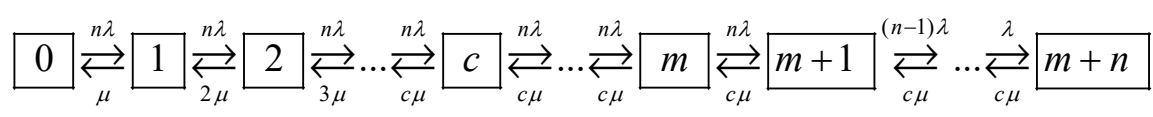

Fig. 1. Graph of system states in the case $1 \leq c \leq m$

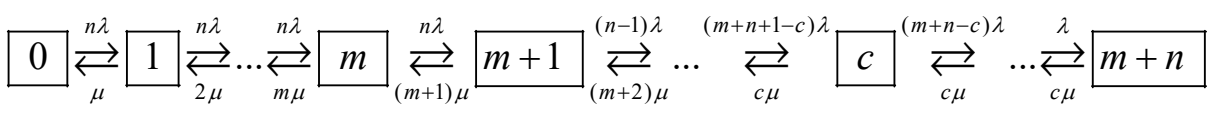

Fig. 2. Graph of system states in the case $m+1 \leq c \leq m+n$

Here $\lambda$ and $\mu$ are the parameters of exponential distributions of the time to failure and the recovery time, respectively. For this system, the time to failure $X_{S}$ and the time between failures $\tilde{X}_{S}$ do not coincide, because the first time interval begins at the time of transition from state " 1 " to state " 0 ", and the second begins at the time of transition from state " $m+1$ " to state " $m$ ". Both states end simultaneously at the moment of transition from the state " $m$ " to the state " $m+1$ ". The downtime $X_{S I}$ is the time spent in a group of states $(m+1, \ldots, m+n)$.

Since the processes, described by the state graphs shown in Figures 1 and 2, are the birth-death processes, we can derive formulas for steady-state probabilities $p_{k}$.

$$
0 \underset{\mu_{1}}{\stackrel{\lambda_{0}}{\rightleftarrows}} 1 \underset{\mu_{2}}{\stackrel{\lambda_{1}}{\rightleftarrows}} 2 \underset{\mu_{3}}{\stackrel{\lambda_{2}}{\rightleftarrows}} \ldots \underset{\mu_{r}}{\stackrel{\lambda_{r-1}}{\rightleftarrows}} r
$$

Fig. 3. Graph of states for the birth-death process

For the birth-death process, described by the state graph shown in Figure 3, the following equations hold:

$$
\lambda_{k} p_{k}=\mu_{k+1} p_{k+1}, \quad p_{k+1}=p_{0} \prod_{i=0}^{k} \frac{\lambda_{i}}{\mu_{i+1}}, \quad 0 \leq k \leq r-1 ; \quad \sum_{k=0}^{r} p_{k}=1 \text {. }
$$

Therefore,

$$
p_{0}=\frac{1}{1+\sum_{k=0}^{r-1} \prod_{i=0}^{k} \frac{\lambda_{i}}{\mu_{i+1}}}, \quad p_{k}=p_{0} \prod_{i=0}^{k-1} \frac{\lambda_{i}}{\mu_{i+1}}, \quad 1 \leq k \leq r
$$

Introducing notation $\rho=\lambda / \mu$, for the case when the number of repair facilities satisfies the condition $1 \leq c \leq m$, we get: 


$$
\begin{aligned}
& p_{0}=\frac{1}{1+\sum_{k=1}^{c} \frac{(n \rho)^{k}}{k !}+\sum_{k=c+1}^{m+1} \frac{(n \rho)^{k}}{c ! c^{k-c}}+\sum_{k=m+2}^{m+n} \frac{n ! n^{m} \rho^{k}}{c !(m+n-k) ! c^{k-c}}} \\
& p_{k}=p_{0} \frac{(n \rho)^{k}}{k !}, \quad 1 \leq k \leq c ; \quad p_{k}=p_{0} \frac{(n \rho)^{k}}{c ! c^{k-c}}, \quad c+1 \leq k \leq m+1 \\
& p_{k}=p_{0} \frac{n ! n^{m} \rho^{k}}{c !(m+n-k) ! c^{k-c}}, \quad m+2 \leq k \leq m+n
\end{aligned}
$$

If $m+2 \leq c \leq m+n$, then

$$
\begin{aligned}
& p_{0}=\frac{1}{1+\sum_{k=1}^{m+1} \frac{(n \rho)^{k}}{k !}+\sum_{k=m+2}^{c} \frac{n ! n^{m} \rho^{k}}{k !(m+n-k) !}+\sum_{k=c+1}^{m+n} \frac{n ! n^{m} \rho^{k}}{c !(m+n-k) ! c^{k-c}}} \\
& p_{k}=p_{0} \frac{(n \rho)^{k}}{k !}, \quad 1 \leq k \leq m+1 ; \quad p_{k}=p_{0} \frac{n ! n^{m} \rho^{k}}{k !(m+n-k) !}, \quad m+2 \leq k \leq c ; \\
& p_{k}=p_{0} \frac{n ! n^{m} \rho^{k}}{c !(m+n-k) ! c^{k-c}}, \quad c+1 \leq k \leq m+n .
\end{aligned}
$$

If $c=m+1$, then

$$
\begin{gathered}
p_{0}=\frac{1}{1+\sum_{k=1}^{m+1} \frac{(n \rho)^{k}}{k !}+\sum_{k=m+2}^{m+n} \frac{n ! n^{m} \rho^{k}}{c !(m+n-k) ! c^{k-c}}} ; \\
p_{k}=p_{0} \frac{(n \rho)^{k}}{k !}, 1 \leq k \leq m+1 ; \quad p_{k}=p_{0} \frac{n ! n^{m} \rho^{k}}{c !(m+n-k) ! c^{k-c}}, \quad m+2 \leq k \leq m+n .
\end{gathered}
$$

Let us introduce notation $T_{k}$ to denote the average time from the moment the system comes to state " $k$ " to the transition to state " $m+1$ ". Then $E\left(X_{S}\right)=T_{0}$, $E\left(\tilde{X}_{S}\right)=T_{m}$.

Suppose that $m+1 \leq c \leq m+n$. Using the properties of exponential distributions, we have

$$
T_{0}=\frac{1}{n \lambda}+T_{1} ; \quad T_{k}=\frac{1+k \mu T_{k-1}+n \lambda T_{k+1}}{n \lambda+k \mu}, 1 \leq k \leq m-1 ; \quad T_{m}=\frac{1+m \mu T_{m-1}}{n \lambda+m \mu} .
$$

Consistently expressing $T_{k}$ through $T_{0}$ for $k \in\{1,2, \ldots, m\}$, we obtain the equalities 


$$
T_{k}=T_{0}-\sum_{i=1}^{k} \frac{k ! \mu^{i-1}}{(k-i) ! i(n \lambda)^{i}}, 1 \leq k \leq m
$$

So we have an equation to find $T_{0}$

$$
\begin{aligned}
T_{m} & =T_{0}-\sum_{i=1}^{m} \frac{m ! \mu^{i-1}}{(m-i) ! i(n \lambda)^{i}}=\frac{1+m \mu T_{m-1}}{n \lambda+m \mu}= \\
& =\frac{1}{n \lambda+m \mu}\left(1+m \mu T_{0}-m \mu \sum_{i=1}^{m-1} \frac{(m-1) ! \mu^{i-1}}{(m-1-i) ! i(n \lambda)^{i}}\right),
\end{aligned}
$$

from whence we obtain

$$
\begin{aligned}
& T_{0}=E\left(X_{S}\right)=\sum_{i=1}^{m+1} \frac{(m+1) ! \mu^{i-1}}{(m+1-i) ! i(n \lambda)^{i}}, \quad T_{m}=E\left(\tilde{X}_{S}\right)=\frac{1}{n \lambda}+\sum_{i=1}^{m} \frac{m ! \mu^{i}}{(m-i) !(n \lambda)^{i+1}}, \\
& T_{k}=T_{0}-\sum_{i=1}^{k} \frac{k ! \mu^{i-1}}{(k-i) ! i(n \lambda)^{i}}=\sum_{i=1}^{m+1} \frac{(m+1) ! \mu^{i-1}}{(m+1-i) ! i(n \lambda)^{i}}-\sum_{i=1}^{k} \frac{k ! \mu^{i-1}}{(k-i) ! i(n \lambda)^{i}}, 1 \leq k \leq m-1 .
\end{aligned}
$$

Thus, we determined not only the mean time to failure and the mean time between failures, but also the average "travel" time $T_{k}$ from state " $\mathrm{k}$ " $(1 \leq k \leq m-1)$ to the moment of system failure.

Let us introduce notation $\tau_{k}$ to denote the average "travel" time from state " $k$ " to state " $\mathrm{m}$ ". Then the mean downtime $E\left(X_{S I}\right)=\tau_{m+1}$. Let us define the mean downtime as the average time spent in a group of states $(m+1, \ldots, m+n)$, assuming that $m+1 \leq c \leq m+n$.

Let $\tilde{p}_{m}$ be the steady-state probability of being in the state " $m$ " for the subsystem, the state graph of which is shown in Figure 4. Given that the average staying time in the state " $m$ " is equal to $1 / n \lambda$, and the probability of being in the group of states $(m+1, \ldots, m+n)$ is equal to $1-\tilde{p}_{m}$, we have

$$
\begin{gathered}
1-\tilde{p}_{m}=\frac{\tau_{m+1}}{\tau_{m+1}+\frac{1}{n \lambda}} \Rightarrow \tau_{m+1}=\frac{1-\tilde{p}_{m}}{n \lambda \tilde{p}_{m}} . \\
m \underbrace{\stackrel{n \lambda}{\rightleftarrows}}_{(m+1) \mu} m+1{\underset{(m+2) \mu}{\rightleftarrows}}_{m}^{\stackrel{(n-1) \lambda}{\rightleftarrows}} \underset{c \mu}{\stackrel{(m+n+1-c) \lambda}{\rightleftarrows}} \stackrel{c}{\underset{c \mu}{\rightleftarrows}} \ldots \underset{c \mu}{\stackrel{(m+n-c) \lambda}{\rightleftarrows}} m+n
\end{gathered}
$$

Fig. 4. Graph of states for the subsystem to determine the mean downtime in the case $m+1 \leq c \leq m+n$ 
Using formulas for stationary probabilities for the birth-death process, described by the state graph shown in Figure 4, we obtain the equality

$$
\frac{1}{\tilde{p}_{m}}=1+\sum_{k=m+1}^{c} \frac{n ! m ! \rho^{k-m}}{k !(m+n-k) !}+\sum_{k=c+1}^{m+n} \frac{n ! m ! \rho^{k-m}}{c !(m+n-k) ! c^{k-c}} .
$$

Therefore, the mean downtime of the system is defined as

$$
\tau_{m+1}=E\left(X_{S I}\right)=\frac{n ! m !}{n \lambda}\left(\sum_{k=m+1}^{c} \frac{\rho^{k-m}}{k !(m+n-k) !}+\sum_{k=c+1}^{m+n} \frac{\rho^{k-m}}{c !(m+n-k) ! c^{k-c}}\right), m+1 \leq c \leq m+n .
$$

In the case when $1 \leq c \leq m$, using the properties of exponential distributions, we have

$$
\begin{aligned}
& T_{0}=\frac{1}{n \lambda}+T_{1} ; \quad T_{k}=\frac{1+k \mu T_{k-1}+n \lambda T_{k+1}}{n \lambda+k \mu}, 1 \leq k \leq c ; \\
& T_{k}=\frac{1+c \mu T_{k-1}+n \lambda T_{k+1}}{n \lambda+c \mu}, c+1 \leq k \leq m-1 ; \quad T_{m}=\frac{1+c \mu T_{m-1}}{n \lambda+c \mu} .
\end{aligned}
$$

Consistently expressing $T_{k}$ through $T_{0}$ for $k \in\{1,2, \ldots, c+1\}$, we obtain the equalities

$$
T_{k}=T_{0}-\sum_{i=1}^{k} \frac{k ! \mu^{i-1}}{(k-i) ! i(n \lambda)^{i}}, 1 \leq k \leq c+1 .
$$

Expressing $T_{k}$ for $k \in\{c+2, \ldots, m\}$ through $T_{c}$ and $T_{c+1}$, we have:

$$
\begin{aligned}
T_{c+k} & =\frac{1}{(n \lambda)^{k-1}}\left(T_{c+1} \sum_{i=1}^{k}(n \lambda)^{k-i}(c \mu)^{i-1}-T_{c} \sum_{i=1}^{k}(n \lambda)^{k-1-i}(c \mu)^{i}-\right. \\
& \left.-\sum_{i=1}^{k-1}(k-i)(n \lambda)^{k-1-i}(c \mu)^{i-1}\right), 2 \leq k \leq m-c .
\end{aligned}
$$

Using relation (16), we can express $T_{m}$ and $T_{m+1}$ through $T_{c}$ and $T_{c+1}$ and, hence, through $T_{0}$. At the same time,

$$
T_{m}=\frac{1+c \mu T_{m-1}}{n \lambda+c \mu} .
$$

Equating the two obtained expressions for $T_{m}$, we obtain an equation for finding $T_{0}$, and we find 


$$
\begin{aligned}
& T_{0}=E\left(X_{S}\right)= \frac{1}{(n \lambda)^{m+1}}\left(\sum_{i=1}^{c+1} \frac{(c+1) !(n \lambda)^{m+1-i} \mu^{i-1}}{(c+1-i) ! i}+\left(\sum_{i=1}^{m-c}(n \lambda)^{m-c-i}(c \mu)^{i}\right) \sum_{i=1}^{c+1} \frac{c !(n \lambda)^{c+1-i} \mu^{i-1}}{(c+1-i) !}+\right. \\
&+\left.\sum_{i=0}^{m-c-1}(m-c-i)(n \lambda)^{m-i}(c \mu)^{i}\right), 1 \leq c \leq m-1 ; \\
& T_{0}=E\left(X_{S}\right)=\frac{1}{(n \lambda)^{c+1}} \sum_{i=1}^{c+1} \frac{(c+1) !(n \lambda)^{c+1-i} \mu^{i-1}}{(c+1-i) ! i}, c=m .
\end{aligned}
$$

By calculating $T_{0}$ and using formulas (15) and (16), we can find not only the mean time between failures $E\left(\tilde{X}_{S}\right)=T_{m}$ but also the average "travel" time $T_{k}$ from state " $k$ " $(1 \leq k \leq m-1)$ to the moment of system failure.

To find the mean downtime $E\left(X_{S I}\right)=\tau_{m+1}$ in the case when $1 \leq c \leq m$, it is sufficient to solve the system of equations

$$
\begin{aligned}
& \tau_{m+1}=\frac{1+(n-1) \lambda \tau_{m+2}}{(n-1) \lambda+c \mu} ; \quad \tau_{m+n}=\frac{1}{c \mu}+\tau_{m+n-1} ; \\
& \tau_{k}=\frac{1+(m+n-k) \lambda \tau_{k-1}+c \mu \tau_{k+1}}{(m+n-k) \lambda+c \mu}, m+2 \leq k \leq m+n-1 .
\end{aligned}
$$

Consistently expressing $\tau_{k}$ through $\tau_{m+n}$ for $k \in\{m+1, m+2, \ldots, m+n-1\}$, we obtain the equalities

$$
\tau_{m+n-k}=\tau_{m+n}-\sum_{i=1}^{k} \frac{k ! \lambda^{i-1}}{(k-i) ! i(c \mu)^{i}(n \lambda)^{i}}, 1 \leq k \leq n-1 .
$$

Equating expressions for $\tau_{m+1}$, obtained from (19) and (20), we have an equation for finding $\tau_{m+n}$ and we find

$$
\tau_{m+n}=\sum_{i=1}^{n} \frac{n ! \lambda^{i-1}}{(n-i) ! i(c \mu)^{i}} .
$$

Substituting the expression for $\tau_{m+n}$ in (20) for $k=n-1$, we obtain the formula for the mean downtime of the system

$$
E\left(X_{S I}\right)=\tau_{m+1}=\sum_{i=1}^{n} \frac{(n-1) ! \lambda^{i-1}}{(n-i) !(c \mu)^{i}}, 1 \leq c \leq m
$$




\section{Conclusions}

This paper shows that the application of Markov reliability models allow us to find stationary indices of the series recoverable system with redundancy and repair facilities for the cases when $1 \leq c \leq m$ and $m+1 \leq c \leq m+n$. We have derived exact formulas for stationary probabilities of system states, a stationary availability coefficient, mean time to failure, mean time between failures, mean downtime, the average "travel" time $T_{k}$ from state " $\mathrm{k}$ " $(1 \leq k \leq m-1)$ to the moment of system failure.

\section{References}

[1] Ushakov, I. (2012). Probabilistic Reliability Models. Hoboken: John Wiley \& Sons.

[2] Zhernovyi, Yu., \& Kopytko, B. (2020). Reliability assessment of a series system with redundancy and repair facilities. J. Appl. Math. Comput. Mech., 19(3), 123-131.

[3] Zhernovyi, Yu., \& Kopytko, B. (2020). Calculating steady-state probabilities of single-channel closed queueing systems using hyperexponential approximation. J. Appl. Math. Comput. Mech., 19(1), 113-120.

[4] Zhernovyi, Yu.V., \& Zhernovyi, K.Yu. (2015). Method of potentials for a closed system with queue length dependent service times. J. of Communications Technology and Electronics, 60(12), 1341-1347.

[5] Aliyev, S.A., Yeleyko, Y.I., \& Zhernovyi, Yu.V. (2019). Calculating steady-state probabilities of closed queueing systems using hyperexponential approximation. Caspian J. of Appl. Math., Economics and Ecology, 7(1), 46-55. 